\title{
氮添加对亚热带山地杜鹃灌丛土壤呼吸的影响
}

\author{
张 蓄 1,2 李家湘 ${ }^{3}$ 谢宗强 $1^{*}$
}

${ }^{1}$ 中国科学院植物研究所植被与环境变化国家重点实验室, 北京 $100093{ }^{2}$ 中国科学院大学, 北京 $100049{ }^{3}$ 中南林业科技大学林学院, 长沙 410004

摘 要 为探究灌从生态系统对大气氮沉降的响应, 2013年1月至2014年9月, 对湖南大围山杜鹃(Rhododendron simsii)灌从群 落进行了短期模拟氮沉降试验, 施氮浓度分别为 $0(\mathrm{CK}) 、 2(\mathrm{LN}) 、 5(\mathrm{MN})$ 和 $10(\mathrm{HN}) \mathrm{g} \cdot \mathrm{m}^{-2} \cdot \mathrm{a}^{-1}$ 。利用 LI-8100土壤碳通量测量系 统测定土壤呼吸速率, 并测定不同氮处理下根系生物量增量和调落物量。结果表明: 该地区土壤呼吸呈现明显的季节动态, 夏季土壤呼吸最强, 冬季最弱。CK、 LN、MN和HN处理样地每年通过土壤呼吸释放的 $\mathrm{CO}_{2}$ 量分别为 $2.37 、 2.79 、 2.26$ 和 $2.30 \mathrm{~kg}$ $\mathrm{CO}_{2} \cdot \mathrm{m}^{-2}$ 。CK、 $\mathrm{LN} 、 \mathrm{MN}$ 和 $\mathrm{HN}$ 处理下, 年平均土壤呼吸速率分别为 $1.71 、 2.01 、 1.63$ 和1.66 $\mu \mathrm{mol} \mathrm{CO}_{2} \cdot \mathrm{m}^{-2} \cdot \mathrm{s}^{-1}, \mathrm{LN}$ 处理样地的 年均土壤呼吸速率与对照样地相比增加了 $17.25 \%, \mathrm{MN}$ 和HN处理则比对照样地稍低。施氮增加了根系生物量增量和调落物量, 但没有达到显著水平。土壤呼吸速率与 $5 \mathrm{~cm}$ 土壤温度呈显著指数相关关系, 与 $5 \mathrm{~cm}$ 土壤的含水量呈显著线性相关关系。CK、 LN、MN和HN处理下, 土壤呼吸的温度敏感性 $\left(Q_{10}\right)$ 值分别为3.96、3.60、3.71和3.51, 表明施氮降低了温度敏感性。氮添加导 致的根系生物量增加是引起该区域土壤呼吸速率变化的一个重要原因。

关键词 土壤呼吸的温度敏感性; 土壤温度; 土壤含水量; 根系生物量; 调落物生物量

引用格式: 张萻, 李家湘, 谢宗强 (2017). 氮添加对亚热带山地杜鹃灌从土壤呼吸的影响. 植物生态学报, 41, 95-104. doi: 10.17521/cjpe.2015.0302

\section{Effects of nitrogen addition on soil respiration of Rhododendron simsii shrubland in the sub- tropical mountainous areas of China}

\author{
ZHANG Qiang $^{1,2}$, LI Jia-Xiang ${ }^{3}$, and XIE Zong-Qiang ${ }^{1 *}$
}

${ }^{1}$ State Key Laboratory of Vegetation and Environment Change, Institute of Botany, Chinese Academy of Sciences, Beijing 100093, China; ${ }^{2}$ University of Chinese Academy of Sciences, Beijing 100049, China; and ${ }^{3}$ Faculty of Forestry, Central South University of Forestry and Technology, Changsha 410004, China

\section{Abstract}

Aims As the second largest C flux between the atmosphere and terrestrial ecosystems, soil respiration plays a vital role in regulating atmosphere $\mathrm{CO}_{2}$ concentration. Therefore, understanding the response of soil respiration to the increasing nitrogen deposition is urgently needed for prediction of future climate change. However, it is still unclear how nitrogen deposition influences soil respiration of shrubland in subtropical China. Our objectives were to explore the effects of different levels of nitrogen fertilization on soil respiration, root biomass increment, and litter biomass, and to analyze the relationships between soil respiration and soil temperature and moisture.

Methods From January 2013 to September 2014, we conducted a short-term simulated nitrogen deposition experiment in the Rhododendron simsii shrubland of Dawei Mountain, located in Hunan Province, southern China. Four levels of nitrogen addition treatments (each level with three replicates) were established: control (CK, no nitrogen addition), low nitrogen addition ( $\mathrm{LN}, 2 \mathrm{~g} \cdot \mathrm{m}^{-2} \cdot \mathrm{a}^{-1}$ ), medium nitrogen addition $\left(\mathrm{MN}, 5 \mathrm{~g} \cdot \mathrm{m}^{-2} \cdot \mathrm{a}^{-1}\right)$ and high nitrogen addition ( $\left.\mathrm{HN}, 10 \mathrm{~g} \cdot \mathrm{m}^{-2} \cdot \mathrm{a}^{-1}\right)$. Soil respiration was measured by LI-8100 soil $\mathrm{CO}_{2}$ efflux system. At the same time, we measured root biomass increment and litter biomass in each plot.

Important findings Soil respiration exhibited a strong seasonal pattern, with the highest rates found in summer and the lowest rates in winter. Annual accumulative soil respiration rate in the CK, LN, MN and HN was $(2.37 \pm$ 0.39), (2.79 \pm 0.42$),(2.26 \pm 0.38)$ and $(2.30 \pm 0.36) \mathrm{kg} \mathrm{CO}_{2} \cdot \mathrm{m}^{-2}$, respectively. Annual mean soil respiration rate in the CK, LN, MN and HN was $(1.71 \pm 0.28),(2.01 \pm 0.30),(1.63 \pm 0.27)$ and $(1.66 \pm 0.26) \mu \mathrm{mol} \mathrm{CO}_{2} \cdot \mathrm{m}^{-2} \cdot \mathrm{s}^{-1}, \mathrm{re}-$ spectively, and it was $17.25 \%$ higher in the LN treatment compared with CK $(p=0.06)$. The root biomass increment was increased by LN, MN, and HN treatments by 18.36\%, 36.49\% and 61.63\%, respectively, compared to CK. The litter biomass was increased by LN, MN, and HN treatments by 35.87\%, 22.17\% and 15.35\%,

收稿日期Received: 2015-08-17 接受日期Accepted: 2016-01-17

* 通信作者Author for correspondence (E-mail: xie@ibcas.ac.cn) 
respectively, compared with CK. Soil respiration exhibited a significant exponential relationship with soil temperature ( $p<0.01, R^{2}$ is 0.77 to 0.82 ) and a significant linear relationship with soil moisture at the depth of $5 \mathrm{~cm}$ ( $p<0.05, R^{2}$ is 0.10 to 0.15 ). The temperature sensitivity $\left(Q_{10}\right)$ value of $\mathrm{CK}, \mathrm{LN}, \mathrm{MN}$ and $\mathrm{HN}$ plots was 3.96, 3.60, 3.71 and 3.51, respectively. These results suggested that nitrogen addition promoted plant growth and decreased the temperature sensitivity of soil respiration. The increase of root biomass under $\mathrm{N}$ addition may be an important reason for the change of soil respiration in the study area.

Key words temperature sensitivity to soil respiration; soil temperature; soil moisture; root biomass; litter biomass

Citation: Zhang Q, Li JX, Xie ZQ (2017). Effects of nitrogen addition on soil respiration of Rhododendron simsii shrubland in the subtropical mountainous areas of China. Chinese Journal of Plant Ecology, 41, 95-104. doi: 10.17521/cjpe.2015.0302

由于化石燃料的燃烧和施肥等人类活动的影 响, 大气氮沉降 $\left(\mathrm{NH}_{4}^{+}{ }_{4} \mathrm{~N}\right.$ 和 $\left.\mathrm{NO}_{3}^{-}-\mathrm{N}\right)$ 在过去的一个世 纪急剧增加(IPCC, 2013)。在亚洲, 由于工农业的快 速发展, 活性氮的使用和释放从1961年的 $14 \mathrm{Tg} \cdot \mathrm{a}^{-1}$ 增加到 2000年的68 $\mathrm{Tg} \cdot \mathrm{a}^{-1}$, 预计到 2030年将达到 $105 \mathrm{Tg} \cdot \mathrm{a}^{-1}$ (Zheng et al., 2002; Denman et al., 2007)。 中国的大气氮沉降也经历了快速的增长, 而且有不 断发展的趋势(Liu et al., 2011), 一些亚热带地区森 林的氮沉降量达到了 30-73 $\mathrm{kg} \cdot \mathrm{hm}^{-2} \cdot \mathrm{a}^{-1}$ (Mo et al., 2006)。低水平的大气氮沉降可以刺激植物的生长, 增加植物对大气中碳的吸收, 从而缓解全球气候变 化(Pregitzer et al., 2008; Thomas et al., 2010)。但是 过度的大气氮沉降会产生许多负面的生态学影响, 比如土壤酸化(Maskell et al., 2010)和生物多样性的 丧失(Högberg et al., 2006)。大气和土壤中的氮素积 累已在很大程度上改变了区域和全球环境, 影响了 陆地生态系统的碳循环(Luo et al., 2006), 从而对未 来的气候变化产生影响(Melillo et al., 2002)。

陆地生态系统的大部分碳储存于土壤中。已有 的研究显示, 全球植物和土壤的碳储量分别为560 Pg (Forster et al., 2007)和>3 300 Pg (Tarnocai et al., 2009)。土壤呼吸是 $\mathrm{CO}_{2}$ 从陆地生态系统返回到大气 中的主要途径(Schlesinger \& Andrews, 2000)。 $\mathrm{CO}_{2}$ 的主要来源是根系和根际微生物呼吸、土壤微生物 和土壤动物呼吸。作为大气和陆地生态系统之间的 第二大碳交换途径, 土壤呼吸每年向大气中释放的 碳量高达68-98 Pg (Raich et al., 2002; BondLamberty \& Thomson, 2010), 在调节大气 $\mathrm{CO}_{2}$ 浓度和 地球的气候变化方面发挥着重要作用。因此, 土壤呼 吸的轻微变化就会对全球的气候和环境产生剧烈的 影响(Davidson \& Janssens, 2006)。导致全球变化的主 要因子(如氮沉降的增加和 $\mathrm{CO}_{2}$ 浓度的上升)在很大程 度上影响了土壤呼吸速率(Bowden et al., 2004; Deng et al., 2010), 因此, 理解全球变化下土壤呼吸的反应 机制对于预测未来的气候变化十分必要。

虽然国内外关于土壤呼吸对氮沉降反应的研究 已有很多, 但由于生物区系类型、环境条件和试验 方法不同, 模拟氮沉降对土壤呼吸的影响至今没有 统一结论(Craine et al., 2001; Moscatelli et al., 2008; Xu \& Wan, 2008)。值得注意的是, 目前关于氮沉降 对土壤呼吸的影响的研究大多是在森林和草原进行 的, 灌丛生态系统作为陆地生态系统的重要组成部 分, 在我国的分布面积仅次于草地而高于森林(胡 会峰等, 2006), 但对其土壤呼吸的研究还较为缺乏, 关于亚热带灌从土壤呼吸的研究更是少见。在灌从 生态系统中, 植被组成不同于森林和草原, 其本底 土壤呼吸值是多少, 对氮沉降如何响应? 这些问题 至今还不清楚。从胡会峰等(2006)对中国主要灌从植 被的研究可以得知, 中国灌丛面积近 $2 \times 10^{8} \mathrm{hm}^{2}$, 约 有一半分布在亚热带区域。而杜鹃(Rhododendron simsii)作为广泛分布的物种, 在我国绝大部分省区均 有生长。基于此, 本研究选择我国亚热带山地杜鹃灌 从作为研究对象, 探讨不同施氮水平下土壤呼吸通 量和季节动态的响应, 并分析土壤呼吸与土壤温度 和土壤含水量的相关关系, 以期为准确地预测环境 变化与土壤呼吸之间的关系提供理论依据。

\section{1 材料和方法}

\section{1 研究区域自然概况}

研究地位于湖南汶阳大围山自然保护区, 地处 罗霄山脉北段, 山脉近东西走向, 以中山地貌为主。 地理位置为 $28.35^{\circ}-28.48^{\circ} \mathrm{N}, 114.03^{\circ}-114.22^{\circ} \mathrm{E}$, 土 壤主要为山地黄棕壤。气候属亚热带季风湿润气候, 年平均气温 $11-16{ }^{\circ} \mathrm{C}$, 无霜期 243 天, 年降水量 1800-2 $000 \mathrm{~mm}$, 年相对湿度高于 $83 \%$ 。植被区划 上, 研究地位于中亚热带常绿阔叶林北部亚地带, 
地带性植被为常绿阔叶林(吴征镒, 1980)。因长期的 人为及自然干扰，尤其是 1958 年成立国有林场后, 大量的砍伐使得森林植被遭到空前的破坏, 直到20 世纪80年代末期, 林场实行封禁管理, 植被才得到 有效恢复(李家湘等, 2015)。现今海拔 $1200 \mathrm{~m}$ 以上的 地带逐渐由灌草从演替成中山山地灌从, 建群种主 要有杜鹃 (Rhododendron simsii)、湖南白檀 (Symplocos paniculata $=$ Symplocos hunanensis, 又称白 檀)、四川冬青(Ilex szechwanensis)、水马桑(Weigela japonica var. sinica $=$ Weigela japonica, 又称日本锦 带花)、圆雉绣球(Hydrangea paniculata)等。其中以 杜鹃为主的灌从最具优势。本试验即选择了海拔 $1400 \mathrm{~m}$ 左右的杜鹃灌从群落作为研究对象, 群落 中杜鹃和湖南白檀的重要值将近 $80 \%$ 。草本层的盖 度约40\%, 其中以短尖薹草(Carex brevicuspis)、香港 双蝴蝶 (Tripterospermum nienkui) 和芒 (Miscanthus sinensis)等占优势(李家湘等, 2015)。试验样地的土 壤理化性质见表1。

\section{2 样地设置}

在湖南汶阳大围山国家森林公园海拔 $1400 \mathrm{~m}$ 左右的位置, 选择分布面积最大的杜鹃灌从设置样 地, 要求地形起伏不大, 保证群落组成一致, 生境 一致。试验采用随机区组设计, 选取 4 个 $5 \mathrm{~m} \times 5 \mathrm{~m}$ 的 小区组成一个区组, 整个试验样地由 3 个区组组成, 小区之间和区组之间的距离分别在 $5 \mathrm{~m}$ 和 $10 \mathrm{~m}$ 左 右。样地四周设置 $10 \mathrm{~m}$ 以上的缓冲带, 避免外界的 干扰。参照该区域的实际氮沉降量(18.23-38.88 $\mathrm{kg} \cdot \mathrm{hm}^{-2} \cdot \mathrm{a}^{-1}$, Lue \& Tian, 2007), 每个区组的4个小区 分别设置对照 $\left(C K, 0 \mathrm{~g} \cdot \mathrm{m}^{-2} \cdot \mathrm{a}^{-1}\right)$ 、低氮 $(\mathrm{LN}, 2$ $\left.\mathrm{g} \cdot \mathrm{m}^{-2} \cdot \mathrm{a}^{-1}\right)$ 、中氮 $\left(M N, 5 \mathrm{~g} \cdot \mathrm{m}^{-2} \cdot \mathrm{a}^{-1}\right)$ 和高氮 $(H N, 10$ $\left.\mathrm{g} \cdot \mathrm{m}^{-2} \cdot \mathrm{a}^{-1}\right) 4$ 种处理, 每种处理 3 个重复。从2012年 8 月起, 在植物生长季节(3-11月)每月施氮1次, 将年 氮添加总量平均分配到每个月。根据不同的养分梯 度, 将 $\mathrm{NH}_{4} \mathrm{NO}_{3}$ 溶于 $20 \mathrm{~L}$ 水中混合均匀, 于晴天的傍
晚用背式喷雾器均匀地喷酒到对应样方林下, 对照 区则喷酒相同量的水, 非生长季有雪覆盖期间不作 任何处理。

\section{3 研究方法}

\subsection{1 土壤温度和含水量的测定}

采用HOBO Pro V2系列温湿度自动记录仪 (HOBO, Onset, USA)采集 $5 \mathrm{~cm}$ 深的土壤温度和含水 量数据, 每 30 min记录1次。

\subsection{2 土壤呼吸的测定}

2012年8月, 在每个测量样方内选取一个 $1 \mathrm{~m} \times$ $1 \mathrm{~m}$ 的小区, 在小区中心安置一个内径 $20 \mathrm{~cm}$ 、高 11 $\mathrm{cm}$ 的PVC土壤呼吸环, PVC环入土 $5 \mathrm{~cm}$ 。每次测量 前一天剪除环内地表活体植物, 以避免对监测数据 产生影响。整个试验期间, 土壤呼吸环的位置保持 不变。2013年1月至2014年9月, 用LI-8100便携式土 壤呼吸测定仪(LI-COR, Nebraska, USA)测量土壤呼 吸。生长季(3-11月)每半个月测量1次, 非生长季在自 然条件允许的情况下每月测量1次(非生长季因自然 条件的限制不能保证每月测量)。每次测量选择晴朗 的天气, 连续测量 3 天, 算出平均值代表当月的土壤 呼吸速率。为了在最大程度上减小日温度变化对土 壤呼吸的影响, 每次测量尽量在10:00-13:00进行。

\subsection{3 灌木层根系生物量增量的测定}

灌木层根系生物量的测量采用数学模拟法(郑 绍伟等, 2007)。2012年8月和2013年8月分别对样方 中的全部灌木进行每木调查, 逐株记录其种名、基 径(地面 $5 \mathrm{~cm}$ 处)和高度。首次测量时, 用红色油漆笔 标记基径的测量位置, 一年后在标记位置进行测 量。对每株灌木编号挂牌, 以便识别。在样方外, 对 优势种杜鹃和湖南白檀分别取 47 株和 30 株标准株, 对非优势种分常绿种和落叶种分别取 168 株和 33 株 标准株。测量标准株的基径和高度, 测量后全根挖 出, 清除所有非根系物质, 对各部分分割后称质量, 并取样(取样100 g)装入布袋带回实验室烘干称量, 以

表1 不同土层的土壤理化性质(平均值土标准误差)

Table 1 Soil physical and chemical properties at different depth (mean $\pm S E$ )

\begin{tabular}{|c|c|c|c|c|c|c|}
\hline $\begin{array}{l}\text { 土壤深度 } \\
\text { Soil depth (cm) }\end{array}$ & $\begin{array}{c}\text { 全碳 } \\
\text { Total C (\%) }\end{array}$ & $\begin{array}{c}\text { 全氮 } \\
\text { Total N (\%) }\end{array}$ & $\mathrm{C:N}$ & $\begin{array}{c}\text { 全磷 } \\
\text { Total } \mathrm{P}\left(\mathrm{mg} \cdot \mathrm{g}^{-1}\right)\end{array}$ & $\mathrm{pH}$ & $\begin{array}{c}\text { 土壤容重 } \\
\text { Soil bulk density }\left(\mathrm{g} \cdot \mathrm{cm}^{-3}\right)\end{array}$ \\
\hline $0-10$ & $4.22 \pm 0.16^{\mathrm{a}}$ & $0.36 \pm 0.01^{\mathrm{a}}$ & $11.63 \pm 0.14^{\mathrm{a}}$ & $0.77 \pm 0.02^{\mathrm{a}}$ & $5.11 \pm 0.01^{b}$ & $0.88 \pm 0.01^{b}$ \\
\hline $10-20$ & $2.72 \pm 0.13^{\mathrm{b}}$ & $0.26 \pm 0.01^{b}$ & $10.42 \pm 0.19^{b}$ & $0.75 \pm 0.02^{\mathrm{a}}$ & $5.17 \pm 0.02^{b}$ & $0.98 \pm 0.02^{\mathrm{a}}$ \\
\hline 20-30 & $2.03 \pm 0.13^{\mathrm{c}}$ & $0.21 \pm 0.01^{\mathrm{c}}$ & $9.48 \pm 0.25^{\mathrm{c}}$ & $0.75 \pm 0.03^{\mathrm{a}}$ & $5.31 \pm 0.03^{\mathrm{a}}$ & $1.01 \pm 0.03^{\mathrm{a}}$ \\
\hline
\end{tabular}

不同字母表示土层间差异显著 $(p<0.05)$ 。

Different letters indicate significant differences among different soil depth $(p<0.05)$. 
构建测量因子与根系生物量之间的关系, 并利用群落 调查的测量因子基径和高度推算样地的根系生物量。 两年测得的根系生物量相减得到根系生物量增量。

\subsection{4 调落物量的测定}

采用面积为 $0.25 \mathrm{~m}^{2}(0.5 \mathrm{~m} \times 0.5 \mathrm{~m})$ 的收集器收 集凋落物, 在每个 $5 \mathrm{~m} \times 5 \mathrm{~m}$ 的样方内随机布设 8 个。 收集从2013年5月到2014年5月产生的调落物, 共收 集5次。收集的调落物在 $65{ }^{\circ} \mathrm{C}$ 恒温下烘至恒质量, 称量得到调落物的质量。对每块样方收集框中的调 落物的质量加和, 得到该样方的调落物年产量。

\subsection{5 数据分析处理}

利用单因素方差分析方法比较不同处理样地之 间根系生物量增量和调落物量的差异。利用重复测 量的方差分析比较不同氮处理下土壤呼吸的差异, 以分析施氮和测定时间及二者的交互作用对杜鹃灌 从土壤呼吸速率的影响。

采用指数模型来拟合土壤呼吸速率 $(R, \mu \mathrm{mol}$ $\left.\mathrm{CO}_{2} \cdot \mathrm{m}^{-2} \cdot \mathrm{s}^{-1}\right)$ 与土壤温度之间的关系: $R=\alpha \mathrm{e}^{\beta T}$ 。其中, $T$ 为 $5 \mathrm{~cm}$ 深土壤温度 $\left({ }^{\circ} \mathrm{C}\right), \alpha$ 为 $0{ }^{\circ} \mathrm{C}$ 时的土壤呼吸速 率, $\beta$ 为土壤呼吸的温度反应系数。土壤呼吸的温度 敏感性 $\left(Q_{10}\right)$ 值由 $\beta$ 值获得: $Q_{10}=\mathrm{e}^{10 \beta}$ 。 $R$ 与 $5 \mathrm{~cm}$ 深土壤 含水量 $(M)$ 的关系采用线性函数来拟合: $R=a M+b$, 其中 $a$ 和 $b$ 是线性回归拟合常数。

用Microsoft Office Excel 2007进行数据整理, 数据的统计分析采用Statistics Analysis System 9.2, 显著水平为 0.05 。动态曲线及相关图形使用Origin 8.5和Microsoft Office Excel 2007软件绘制。

\section{2 结果}

\section{1 土壤温度和含水量的季节变化}

观测结果表明, 地下 $5 \mathrm{~cm}$ 土壤温度具有明显的 季节变化, 在1月份达到最低((4.18 \pm 0.35$\left.){ }^{\circ} \mathrm{C}\right)$, 之 后开始上升, 在8月份达到最高((20.62 \pm 0.36$\left.){ }^{\circ} \mathrm{C}\right)$ (图1)。地下 $5 \mathrm{~cm}$ 土壤含水量比较稳定, 季节变化不 是很明显, 波动范围为 $0.15 \%-0.28 \%$, 但秋季的土 壤含水量相对较低。本试验地点2013年的年平均土 壤温度为 $(14.17 \pm 0.30){ }^{\circ} \mathrm{C}$, 年平均土壤湿度为 $(0.23 \pm 0.06) \mathrm{cm}^{3} \cdot \mathrm{cm}^{-3}$ 。

\section{2 氮添加对根系生物量增量和凋落物量的影响}

经过 1 年的施氮处理, 4 种不同氮添加样地的根 系生物量增量分别为734.05、868.84、1 001.91和 $1186.50 \mathrm{~kg} \cdot \mathrm{hm}^{-2}$ (图2A)。LN、MN和HN处理样地

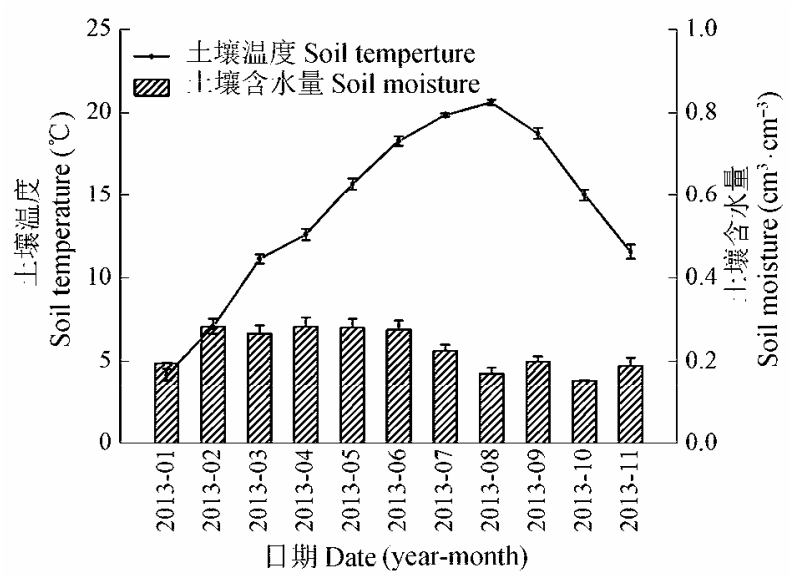

图1 土壤温度和含水量的季节变化(平均值土标准误差)。

Fig. 1 Seasonal variations of soil temperature and moisture (mean $\pm S E$ ).
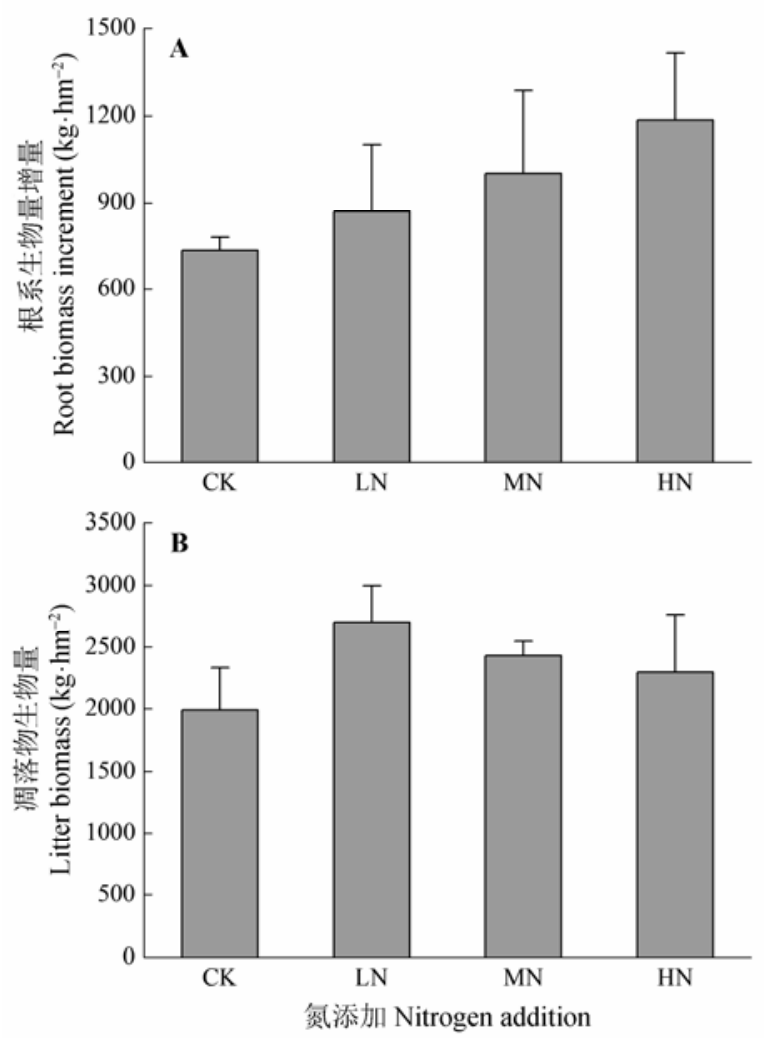

图2 不同氮处理下的根系生物量增量(A)和调落物生物量 (B) (平均值 \pm 标准误差)。CK、LN、MN和 $\mathrm{HN}$ 为 4 种施氮水平, 分别代表氮添加浓度为 $0 、 2 、 5$ 和 $10 \mathrm{~g} \cdot \mathrm{m}^{-2} \cdot \mathrm{a}^{-1}$ 。

Fig. 2 Root biomass increment (A) and litter biomass (B) under different nitrogen treatments (mean $\pm S E$ ). CK, LN, MN and $\mathrm{HN}$ stand for $0,2,5$ and $10 \mathrm{~g} \cdot \mathrm{m}^{-2} \cdot \mathrm{a}^{-1}$ nitrogen addition, respectively.

的根系生物量增量分别比对照样地高出 $18.36 \%$ 、 $36.49 \%$ 和 $61.63 \%$ ，说明氮添加对植物根系的生长有 促进作用, 并且氮添加浓度越高, 促进作用越明显。 
单因素方差分析结果显示各处理之间的差异未达到 显著水平 $(p=0.53)$ 。

收集从2013年5月到2014年5月产生的调落物, 烘干后测其干质量, 4种处理样地的调落物产量分别 为1 989.40、2 703.09、2 430.36和2 $294.71 \mathrm{~kg} \cdot \mathrm{hm}^{-2}$ (图2B)。LN、MN和HN处理样地的调落物量分别比 对照样地高出 $35.87 \% 、 22.17 \%$ 和15.35\%, 表明氮添 加处理增加了调落物产量。单因素方差分析结果显 示各处理之间的差异不显著 $(p=0.52)$ 。

\section{3 氮添加对土壤呼吸季节动态和通量的影响}

在2013年1-11月和2014年3-9月, CK、LN、MN 和HN处理下土壤呼吸的月平均速率均呈现出明显 的季节动态, 且变化趋势相同。夏季7、8月份土壤 呼吸速率达到最高(最大值分别为3.17、3.49、2.71 和3.09 $\mu \mathrm{mol} \mathrm{CO}_{2} \cdot \mathrm{m}^{-2} \cdot \mathrm{s}^{-1}$ ), 此时各处理之间的差异 较明显; 冬季11月至次年1月土壤呼吸速率达到最 低 (最小值分别为 $0.44 、 0.65 、 0.54$ 和 $0.55 \mu \mathrm{mol}$ $\mathrm{CO}_{2} \cdot \mathrm{m}^{-2} \cdot \mathrm{s}^{-1}$ ), 此时各处理之间的差异较小(图3)。 $\mathrm{LN}$ 处理样地的各月土壤呼吸速率均高于其他样地。

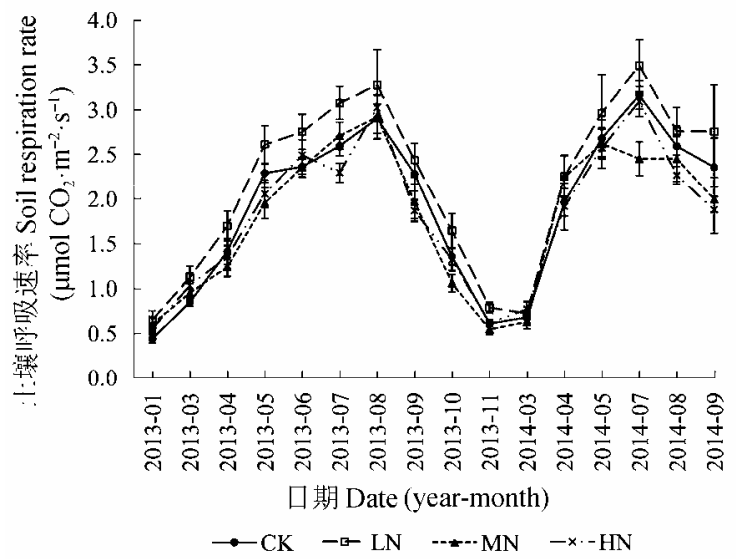

图3 不同氮添加浓度下土壤呼吸的季节动态(平均值士标准 误差)。CK、LN、MN和HN为4种施氮水平, 分别代表的氮 添加浓度为 $0 、 2 、 5$ 和 $10 \mathrm{~g} \cdot \mathrm{m}^{-2} \cdot \mathrm{a}^{-1}$ 。

Fig. 3 Seasonal variations of soil respiration under different nitrogen treatments (mean $\pm S E$ ). $\mathrm{CK}, \mathrm{LN}, \mathrm{MN}$ and $\mathrm{HN}$ stand for $0,2,5$ and $10 \mathrm{~g} \cdot \mathrm{m}^{-2} \cdot \mathrm{a}^{-1}$ nitrogen addition, respectively.

根据对照样地2013年的土壤呼吸值计算, 研究 区域年平均土壤呼吸速率为 $(1.71 \pm 0.28) \mu \mathrm{mol}$ $\mathrm{CO}_{2} \cdot \mathrm{m}^{-2} \cdot \mathrm{s}^{-1}$, 相当于每年释放 $\mathrm{CO}_{2}(2.37 \pm 0.39)$ $\mathrm{kg} \cdot \mathrm{m}^{-2}$ 。4种氮处理中, LN处理的土壤呼吸速率在 2013年生长季和2014年生长季均高于对照样地, $\mathrm{CO}_{2}$ 排放量分别增加了15.51\%和11.25\% (图4)。 $\mathrm{MN}$

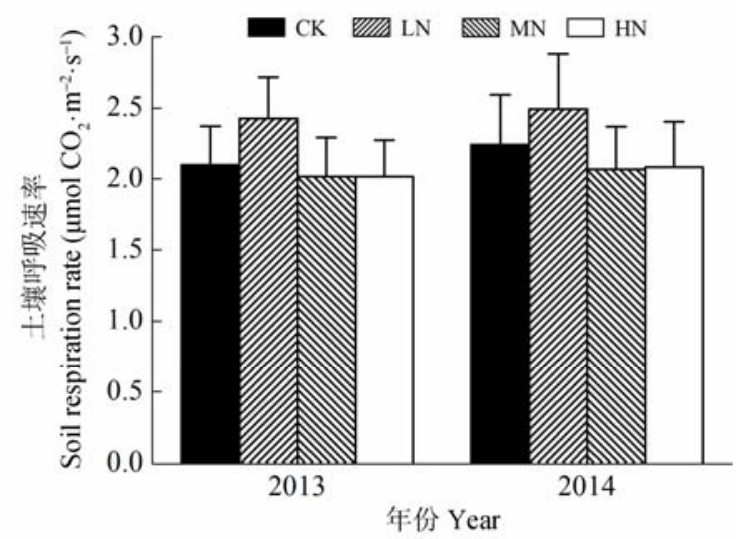

图4 不同氮添加浓度下的生长季土壤呼吸速率(平均值土标 准误差)。CK、 LN、MN和 $\mathrm{HN}$ 为4种施氮水平, 分别代表的 氮添加浓度为 $0 、 2 、 5$ 和 $10 \mathrm{~g} \cdot \mathrm{m}^{-2} \cdot \mathrm{a}^{-1}$ 。

Fig. 4 Soil respiration rate in the growing season under different nitrogen treatments (mean $\pm S E$ ). $\mathrm{CK}, \mathrm{LN}, \mathrm{MN}$ and $\mathrm{HN}$ stand for $0,2,5$ and $10 \mathrm{~g} \cdot \mathrm{m}^{-2} \cdot \mathrm{a}^{-1}$ nitrogen addition, respectively.

和HN处理的土壤呼吸比对照样地低。进行重复测量 的方差分析显示, 氮处理对土壤呼吸的影响在2013 年全年和2013年生长季均接近显著水平 $(p$ 值分别为 0.06 和 0.08$)$, 在 2014 年生长季不显著 $(p=0.27)$ 。测 定时间对土壤呼吸产生显著影响 $(p<0.01)$, 而测定 时间和氮添加的交互作用并不显著(2013和2014年 生长季的 $p$ 值分别为 0.73 和 0.58 )。

\section{4 土壤呼吸与土壤温度和土壤含水量的关系}

不同氮处理下, 杜鹃灌从的土壤呼吸与 $5 \mathrm{~cm}$ 深 土壤温度均呈极显著的指数相关关系 $(p<0.01)$ (图 $5)$, 土壤温度可以解释土壤呼吸变化的 $76.53 \%-$ $82.21 \%, C K 、 L N 、 M N$ 和HN处理下的 $Q_{10}$ 值分别为 3.96、3.60、3.71和3.51。与对照样地相比, 施氮降 低了 $Q_{10}$ 值。土壤呼吸与 $5 \mathrm{~cm}$ 深土壤含水量呈显著线 性相关关系 $(p<0.05)$ (图6), 土壤含水量可以解释土 壤呼吸变化的 $9.93 \%-15.21 \%$ 。与土壤温度相比, 土 壤含水量对土壤呼吸变化的解释率较低。氮处理下 土壤温度与土壤呼吸的相关性与对照相比有所下 降, 而土壤含水量与土壤呼吸的相关性与对照相比 有上升的趋势。

\section{3 讨论}

\section{1 施氮对根系生物量增量和凋落物量的影响}

氮添加对根系生物量增量和调落物产量的影响 在一定程度上反映了其对植物群落生产力的影响。 大量研究显示氮沉降对陆地生态系统的植物生长有 促进作用(Högberg, 2007; Hyvonen et al., 2008)。 


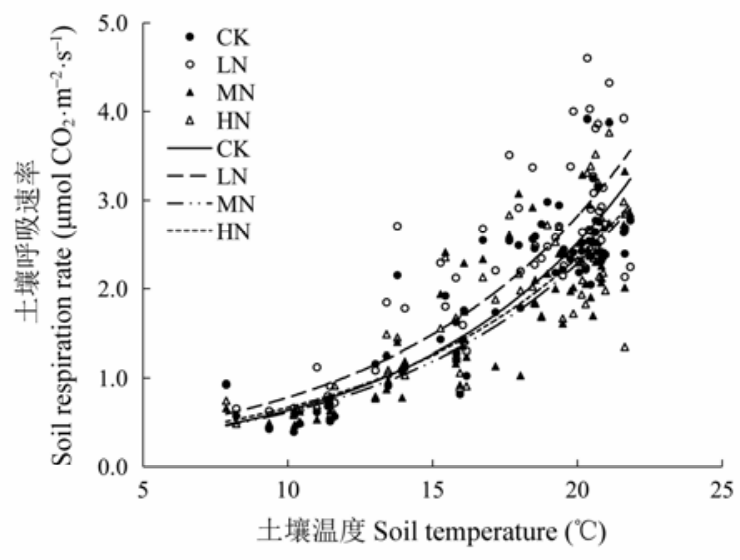

CK $y=0.161 \mathrm{e}^{0.138 x}, R^{2}=0.82, p<0.01, Q_{10}=3.96$ LN $y=0.217 \mathrm{e}^{0.128 x}, R^{2}=0.77, p<0.01, Q_{10}=3.60$ MN $y=0.166 \mathrm{e}^{0.131 x}, R^{2}=0.80, p<0.01, Q_{10}=3.71$ HN $y=0.190 \mathrm{e}^{0.126 x}, R^{2}=0.81, p<0.01, Q_{10}=3.51$

图5 不同氮添加水平下 $5 \mathrm{~cm}$ 深度土壤呼吸与土壤温度的关 系。CK、LN、MN和HN为 4 种施氮水平, 分别代表的氮添加 浓度为 $0 、 2 、 5$ 和 $10 \mathrm{~g} \cdot \mathrm{m}^{-2} \cdot \mathrm{a}^{-1}$ 。 $Q_{10}$, 土壤呼吸的温度敏感性。 Fig. 5 Relationships between soil respiration rate and soil temperature at $5 \mathrm{~cm}$ soil depth under different nitrogen treatments. CK, LN, MN and HN stand for $0,2,5$ and $10 \mathrm{~g} \cdot \mathrm{m}^{-2} \cdot \mathrm{a}^{-1}$ nitrogen addition, respectively. $Q_{10}$, temperature sensitivity to soil respiration.

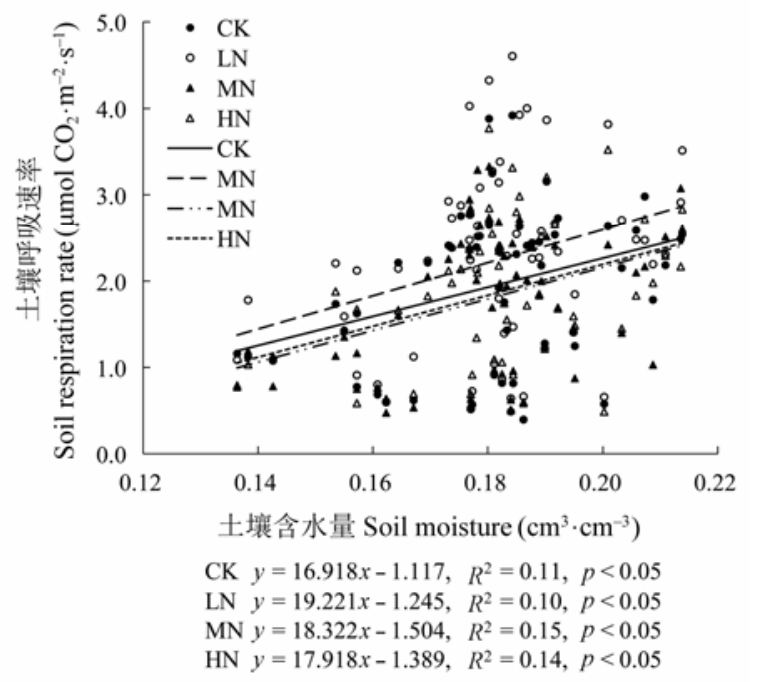

图6 不同氮添加水平下 $5 \mathrm{~cm}$ 深度土壤呼吸与土壤含水量的 关系。CK、LN、MN和HN为 4 种施氮水平, 分别代表的氮添 加浓度为 $0 、 2 、 5$ 和 $10 \mathrm{~g} \cdot \mathrm{m}^{-2} \cdot \mathrm{a}^{-1}$ 。

Fig. 6 Relationships between soil respiration rate and soil moisture at $5 \mathrm{~cm}$ soil depth under different nitrogen treatments. CK, LN, MN and HN stand for 0, 2, 5 and $10 \mathrm{~g} \cdot \mathrm{m}^{-2} \cdot \mathrm{a}^{-1}$ nitrogen addition, respectively.

LeBauer和Treseder (2008)对126个氮添加实验进行 整合分析的结果表明, 大多数陆地生态系统生产力 受到氮素的限制, 且不同生态系统类型受氮素限制 的程度不同, 因此氮添加对生产力的促进程度也不
同。本研究结果显示, 氮添加对杜鹃灌从群落的根 系生长和地上调落物的产生都有促进作用(图2), 但 没有达到显著水平，这可能有以下两个原因: 1)本 研究区域 $0-10 \mathrm{~cm}$ 的土壤氮含量为 $0.36 \%$ (表 1 ), 且 氮沉降处于较高水平 $\left(27.06 \mathrm{~kg} \cdot \mathrm{hm}^{-2} \cdot \mathrm{a}^{-1}\right.$, Lue \& Tian, 2007), 氮不是植物生长的主要限制因素, 进行氮添 加对植物生长的促进作用有限; 2)土壤中其他养分 的限制会导致植物生长对氮添加的响应减弱, 比如 在氮输入不断增加的情况下, 磷会成为限制生长的 主要因素(Elser et al., 2009); 随着土壤氮含量的增 加, 还会出现碳限制的情况(Aber et al., 1989)。

\section{2 施氮对杜鹃灌丛土壤呼吸的影响}

研究结果显示, 不同氮处理下的土壤呼吸呈现 出相似的季节变化模式, 在夏季达到最大, 冬季降 到最低, 这与在中国亚热带其他地区得到的研究结 果相符(Tang et al., 2006; Mo et al., 2007)。土壤呼吸 的季节变化主要受到温度等气候因子和植物生长的 调控影响(韩广轩和周广胜, 2009)。2013年在对照样 地测得的杜鹃灌从年 $\mathrm{CO}_{2}$ 释放量为 $(2.37 \pm 0.39)$ $\mathrm{kg} \cdot \mathrm{m}^{-2}$, 低于Deng等(2010)在中国南亚热带森林测 得的值((3.53 \pm 0.03$\left.) \mathrm{kg} \cdot \mathrm{m}^{-2}\right)$, 但高于中国暖温带森 林的年 $\mathrm{CO}_{2}$ 释放量 $\left((1.50 \pm 0.03) \mathrm{kg} \cdot \mathrm{m}^{-2}\right.$, Sun et al., 2014)。试验结果显示, 低氮处理对土壤呼吸有促进 作用，而中氮和高氮处理则有轻微的抑制效应。综 合前人的研究可知, 研究区域和施氮浓度不同, 土 壤呼吸的响应也不同, 这受到土壤本身养分状况的 影响(Mo et al., 2007)。在氮含量丰富的环境中氮添 加会抑制土壤呼吸, 而在氮限制的地区氮添加则会 促进土壤呼吸(Hyvonen et al., 2007; Cusack et al., 2011)。

一般来说, 将土壤呼吸分为自养呼吸(根系和 根际微生物呼吸)和异养呼吸(微生物和土壤动物呼 吸)两个组分(Sotta et al., 2004), 土壤总呼吸对氮添 加的反应是自养呼吸、异养呼吸反应的综合效应。 在全球范围内的森林生态系统中, 自养呼吸约占土 壤总呼吸的45.8\% (Hanson et al., 2000)。Zhou等 (2014)对来自不同生态系统的295个研究结果进行 整合分析, 结果显示自养呼吸对氮添加的反应与根 系生物量密切相关 $\left(R^{2}=0.803\right)$ 。 Rodeghiero和 Cescatti (2006)对来自7种不同森林生态系统的数据 进行研究, 也发现土壤呼吸与根系生物量之间存在 着线性回归关系。所以, 氮添加对根系生物量的影 
响将会很大程度上影响植物根呼吸和与之相关的根 际微生物呼吸。本试验中, LN、MN和HN处理样地 的根系生物量年增量与对照样地相比, 分别高出了 $18.36 \% 、 36.49 \%$ 和 $61.63 \%$ 。可以看出, 随着氮添加 浓度的升高, 根系生物量年增量呈现出递增的趋势, 因此会导致植物自养呼吸也随之上升。土壤异养呼 吸来源于土壤中有机物质的分解, 主要依赖于分解 底物的质量和数量、胞外酶活性、微生物生物量和 微生物活性(Ryan \& Law, 2005)。Janssens等(2010) 和Zhou等(2014)利用大量研究结果进行的整合分析 显示, 氮添加与异养呼吸呈负相关关系, 特别是在 生产力高、氮素不受限制的生态系统中, 这种负相 关性更明显。Egerton-Warburton和Allen (2000)在加 利福尼亚沿海灌从林中进行的试验表明, 氮肥会降 低菌根感染率和存活率。Rühling和Tyler (1991)观察 到氮添加会使菌根真菌几乎停止产生子实体。研究 发现, 在目前的氮添加浓度(通常大于 $5 \mathrm{~kg} \cdot \mathrm{hm}^{-2} \cdot \mathrm{a}^{-1}$ ) 下, 调落物的分解经常是被抑制的(Knorr et al., 2005), 主要是因为腐生性营养的微生物群落组成 的改变(Gallo et al., 2004)和酶活性的改变(Frey et al., 2004)。在本研究中, 作为微生物分解的底物, 调 落物在氮处理样地中的生物量要高于对照样地(图 3), 分别比对照样地高出 $35.87 \% 、 22.17 \%$ 和 $15.35 \%$ 。 但是, 氮添加在使地上部分调落物增加的同时, 也 可能导致微生物生物量和活性的下降, 从而在一定 程度上限制调落物的分解(Burton et al., 2004), 这也 许会使得异养呼吸有所降低。另外, 有研究表明, 随 着施氮时间的增加, 氮添加对调落物分解的抑制作 用也会增强(Fang et al., 2007)。综上可知, 自养呼吸 和异养呼吸对氮添加的不同反应综合导致了土壤总 呼吸对氮添加的响应。

\section{3 不同氮处理下土壤呼吸与土壤温度和土壤含 水量的关系}

在本研究区域, 土壤呼吸与土壤温度 $\left(R^{2}\right.$ 为 $0.77-0.82, p<0.001)$ 和土壤水分 $\left(R^{2}\right.$ 为 $0.10-0.15, p<$ 0.05) 显著正相关, 这一结果与已有的报道一致 (Tang et al., 2006; Mo et al., 2007, 2008)。土壤水分 与土壤呼吸的相关性要小于土壤温度, 说明本区域 土壤温度是影响土壤呼吸变化的主导因素, 这与研 究地的亚热带季风湿润气候类型有关, 本地区温度 的季节变化明显, 降水丰富, 土壤含水量较高, 不 是土壤呼吸的主要限制因子。在氮处理下土壤呼吸
与土壤含水量的相关性与对照相比有所上升, 我们 推测这是由于氮添加对植物生长的促进会加强植物 对土壤水分的消耗(段洪浪等, 2009), 同时氮添加会 增加叶片扩散导度和气孔导度(Deng et al., 2010), 加剧了水分的散失, 从而降低土壤含水量。当土壤 水分含量降低时, 土壤含水量与土壤呼吸的相关性 会更高(Dilustro et al., 2005)。

作为反映土壤呼吸与土壤温度间关系的重要指 标, $Q_{10}$ 值反映了土壤呼吸的温度敏感性, 对于量化 和预测未来气候变化下的全球碳循环具有重要意义 (Davidson \& Janssens, 2006)。本试验对照样地的 $Q_{10}$ 值为 3.96, 高于前人测得的灌从生态系统的 $Q_{10}$ 值 (2.49 \pm 0.54 , Peng et al., 2009)。这可能与本研究区域 海拔较高有关。罗璐等(2011)对同一地区不同海拔 梯度上的4种典型森林的土壤呼吸的研究显示, 海 拔越高, 土壤呼吸对温度变化的响应越敏感。本研 究中, 氮添加使得 $Q_{10}$ 值与对照相比有所降低, 这与 前人的研究结果(Mo et al., 2008; Tu et al., 2013; Sun et al., 2014)相似。一个可能的原因是氮添加通过形 成难利用有机质增强了土壤有机碳的稳定性, 氮与 土壤有机质结合会产生高度耐微生物酶降解的化合 物(Janssens et al., 2010), 使得微生物异养呼吸的底 物可利用性降低。呼吸底物的可利用性在土壤呼吸 对土壤温度的响应中起关键作用, 当底物供应下降 时, 土壤呼吸的温度敏感性也会下降(Deng et al., 2010)。同时氮添加也会降低微生物酶活性(Ma et al., 2014)。这些改变会降低分解作用对温度变化的敏感 性, 从而导致土壤呼吸的 $Q_{10}$ 值降低。但是也有研究 得出了相反的结论, 即氮添加增强了土壤呼吸的温 度敏感性(Pendall et al., 2004; Deng et al., 2010)。这 也许同样与底物供应有关, 在养分贫痊的地区, 施 氮促进植物生长, 增加了碳向根系的分配(Deng et al., 2010), 使得施氮对土壤有机质含量的促进作用 大于其抑制作用, 从而增加了底物供应, 使 $Q_{10}$ 值增 加。总的来说, 氮沉降对 $Q_{10}$ 的影响是个复杂的过程, 受到生态系统类型、环境因子和土壤养分含量等多 种因素的影响(Zheng et al., 2009), 要综合考虑各因 素的交互作用, 才能准确判断全球变化背景下氮沉 降对 $Q_{10}$ 的影响。

基金项目 中国科学院战略性先导科技专项(XDA05050302)。 
致谢 感谢中南林业科技大学林学院的老师和学生 在样品采集、样品处理和野外监测工作中给予的帮 助。

\section{参考文献}

Aber JD, Nadelhoffer KJ, Steudler P, Melillo JM (1989). Nitrogen saturation in northern forest ecosystems. Bioscience, 39, 378-386.

Bond-Lamberty B, Thomson A (2010). A global database of soil respiration data. Biogeosciences, 7, 1915-1926.

Bowden RD, Davidson E, Savage K, Arabia C, Steudler P (2004). Chronic nitrogen additions reduce total soil respiration and microbial respiration in temperate forest soils at the Harvard Forest. Forest Ecology and Management, 196, 43-56.

Burton AJ, Pregitzer KS, Crawford JN, Zogg GP, Zak DR (2004). Simulated chronic $\mathrm{NO}_{3}{ }^{-}$deposition reduces soil respiration in northern hardwood forests. Global Change Biology, 10, 1080-1091.

Craine JM, Wedin DA, Reich PB (2001). Grassland species effects on soil $\mathrm{CO}_{2}$ flux track the effects of elevated $\mathrm{CO}_{2}$ and nitrogen. New Phytologist, 150, 425-434.

Cusack DF, Silver WL, Torn MS, McDowell WH (2011). Effects of nitrogen additions on above- and below-ground carbon dynamics in two tropical forests. Biogeochemistry, 104, 203-225.

Davidson EA, Janssens IA (2006). Temperature sensitivity of soil carbon decomposition and feedbacks to climate change. Nature, 440, 165-173.

Denman KL, Brasseur G, Chidthaisong A, Ciais P, Cox PM, Dickinson RE, Hauglustaine D, Heinze C, Holland E, Jacob D, Lohmann U, Ramachandran S, da Silva Dias PL, Wofsy SC, Zhang X (2007). Couplings between changes in the climate system and biogeochemistry. In: Solomon S, Qin D, Manning M, Marquis M, Averyt K, Tignor MMB, Miller HLR, Chen Z eds. Climate Change 2007, The Physical Science Basis. Contribution of Working Group I to the Fourth Assessment Report of the Intergovernmental Panel on Climate Change. Cambridge University Press, Cambridge, UK.

Deng Q, Zhou G, Liu J, Liu S, Duan H, Zhang D (2010). Responses of soil respiration to elevated carbon dioxide and nitrogen addition in young subtropical forest ecosystems in China. Biogeosciences, 7, 315-328.

Dilustro JJ, Collins B, Duncan L, Crawford C (2005). Moisture and soil texture effects on soil $\mathrm{CO}_{2}$ efflux components in southeastern mixed pine forests. Forest Ecology and Management, 204, 85-95.

Duan HL, Liu JX, Deng Q, Chen XM, Zhang DQ (2009). Effects of elevated $\mathrm{CO}_{2}$ and $\mathrm{N}$ deposition on plant biomass accumulation and allocation in subtropical forest ecosystems: A mesocosm study. Journal of Plant Ecology (Chinese Version), 33, 570-579. (in Chinese with English ab- stract) [段洪浪, 刘菊秀, 邓琦, 陈小梅, 张德强 (2009). $\mathrm{CO}_{2}$ 浓度升高与氮沉降对南亚热带森林生态系统植物 生物量积累及分配格局的影响. 植物生态学报, 33, 570-579.]

Egerton-Warburton L, Allen E (2000). Shifts in the diversity of arbuscular mycorrhizal fungi along an anthropogenic nitrogen gradient. Ecological Applications, 10, 484-496.

Elser JJ, Andersen T, Baron JS, Bergstroem A-K, Jansson M, Kyle M, Nydick KR, Steger L, Hessen DO (2009). Shifts in lake $\mathrm{N}: \mathrm{P}$ stoichiometry and nutrient limitation driven by atmospheric nitrogen deposition. Science, 326, 835-837.

Fang H, Mo JM, Peng SL, Li ZA, Wang H (2007). Cumulative effects of nitrogen additions on litter decomposition in three tropical forests in southern China. Plant and Soil, 297, 233-242.

Forster P, Ramaswamy V, Artaxo P, Berntsen T, Betts R, Fahey DW, Haywood J, Lean J, Lowe DC, Myhre G, Nganga J, Prinn R, Raga G, Schulz M, van Dorland R (2007). Changes in atmospheric constituents and in radiative forcing. In: Solomon S, Qin D, Manning M, Marquis M, Averyt K, Tignor MMB, Miller HLR, Chen Z eds. Climate Change 2007, The Physical Science Basis. Contribution of Working Group I to the Fourth Assessment Report of the Intergovernmental Panel on Climate Change. Cambridge University Press, Cambridge, UK.

Frey SD, Knorr M, Parrent JL, Simpson RT (2004). Chronic nitrogen enrichment affects the structure and function of the soil microbial community in temperate hardwood and pine forests. Forest Ecology and Management, 196, 159-171.

Gallo M, Amonette R, Lauber C, Sinsabaugh RL, Zak DR (2004). Microbial community structure and oxidative enzyme activity in nitrogen-amended north temperate forest soils. Microbial Ecology, 48, 218-229.

Han GX, Zhou GS (2009). Review of spatial and temporal variations of soil respiration and driving mechanisms. Chinese Journal of Plant Ecology, 33, 197-205. (in Chinese with English abstract) [韩广轩, 周广胜 (2009). 土 壤呼吸作用时空动态变化及其影响机制研究与展望. 植物生态学报, 33, 197-205.]

Hanson PJ, Edwards NT, Garten CT, Andrews JA (2000). Separating root and soil microbial contributions to soil respiration: A review of methods and observations. Biogeochemistry, 48, 115-146.

Högberg P (2007). Environmental science: Nitrogen impacts on forest carbon. Nature, 447, 781-782.

Högberg P, Fan HB, Quist M, Binkley D, Tamm CO (2006). Tree growth and soil acidification in response to 30 years of experimental nitrogen loading on boreal forest. Global Change Biology, 12, 489-499.

Hu HF, Wang ZH, Liu GH, Fu BJ (2006). Vegetation carbon storage of major shrublands in China. Journal of Plant Ecology (Chinese Version), 30, 539-544. (in Chinese with

www.plant-ecology.com 
English abstract) [胡会峰, 王志恒, 刘国华, 傅伯杰 (2006). 中国主要灌丛植被碳储量. 植物生态学报，30, 539-544.]

Hyvonen R, Agren GI, Linder S, Persson T, Cotrufo MF, Ekblad A, Freeman M, Grelle A, Janssens IA, Jarvis PG, Kellomaki S, Lindroth A, Loustau D, Lundmark T, Norby RJ, Oren R, Pilegaard K, Ryan MG, Sigurdsson BD, Stromgren M, van Oijen M, Wallin G (2007). The likely impact of elevated $\mathrm{CO}_{2}$, nitrogen deposition, increased temperature and management on carbon sequestration in temperate and boreal forest ecosystems: A literature review. New Phytologist, 173, 463-480.

Hyvonen R, Persson T, Andersson S, Olsson B, Agren GI, Linder $S$ (2008). Impact of long-term nitrogen addition on carbon stocks in trees and soils in northern Europe. Biogeochemistry, 89, 121-137.

IPCC (Intergovernmental Panel on Climate Change) (2013). Contribution of working group 1 to the fifth assessment report of the intergovernmental panel on climate change. In: Stocker TF, Qin DH, Plattner GK, Tignor MMB, Allen SK, Boschung J, Nauels A, Xia Y, Bex V, Midgley PM eds. Climate Change 2013: The Physical Science Basis. Cambridge University Press, Cambridge, UK.

Janssens IA, Dieleman W, Luyssaert S, Subke JA, Reichstein M, Ceulemans R, Ciais P, Dolman AJ, Grace J, Matteucci G, Papale D, Piao SL, Schulze ED, Tang J, Law BE (2010). Reduction of forest soil respiration in response to nitrogen deposition. Nature Geoscience, 3, 315-322.

Knorr M, Frey SD, Curtis PS (2005). Nitrogen additions and litter decomposition: A meta-analysis. Ecology, 86, 32523257.

LeBauer DS, Treseder KK (2008). Nitrogen limitation of net primary productivity in terrestrial ecosystems is globally distributed. Ecology, 89, 371-379.

Li JX, Zhang X, Xie ZQ, Lu CF, Tu XY, Xun Y (2015). Community composition and structure of Rhododendron simsii shrubland in the Dawei Mountain, Hunan Province. Biodiversity Science, 23, 815-823. (in Chinese with English abstract) [李家湘, 张旭, 谢宗强, 卢从发, 涂向阳, 寻 院 (2015). 湖南大围山杜鹃灌从的群落组成及结构特 征. 生物多样性, 23, 815-823.]

Liu X, Duan L, Mo J, Du E, Shen J, Lu X, Zhang Y, Zhou X, He C, Zhang F (2011). Nitrogen deposition and its ecological impact in China: An overview. Environmental Pollution, 159, 2251-2264.

Lue CQ, Tian HQ (2007). Spatial and temporal patterns of nitrogen deposition in China: Synthesis of observational data. Journal of Geophysical Research-Atmospheres, 112, D22S05. doi: 10.1029/2006JD007990.

Luo L, Sheng GZ, Xie ZQ, Zhou LG (2011). Components of soil respiration and its temperature sensitivity in four types of forests along an elevational gradient in Shennongjia,
China. Chinese Journal of Plant Ecology, 35, 722-730. (in Chinese with English abstract) [罗璐, 申国珍, 谢宗强, 周利光 (2011). 神农架海拔梯度上4种典型森林的土壤 呼吸组分及其对温度的敏感性. 植物生态学报, 35, 722-730.]

Luo YQ, Hui DF, Zhang DQ (2006). Elevated $\mathrm{CO}_{2}$ stimulates net accumulations of carbon and nitrogen in land ecosystems: A meta-analysis. Ecology, 87, 53-63.

Ma YC, Zhu B, Sun ZZ, Zhao C, Yang Y, Piao SL (2014). The effects of simulated nitrogen deposition on extracellular enzyme activities of litter and soil among different-aged stands of larch. Journal of Plant Ecology, 7, 240-249.

Maskell LC, Smart SM, Bullock JM, Thompson K, Stevens CJ (2010). Nitrogen deposition causes widespread loss of species richness in British habitats. Global Change Biology, 16, 671-679.

Melillo JM, Steudler PA, Aber JD, Newkirk K, Lux H, Bowles FP, Catricala C, Magill A, Ahrens T, Morrisseau S (2002). Soil warming and carbon-cycle feedbacks to the climate system. Science, 298, 2173-2176.

Mo JM, Brown S, Xue JH, Fang YT, Li ZA (2006). Response of litter decomposition to simulated $\mathrm{N}$ deposition in disturbed, rehabilitated and mature forests in subtropical China. Plant and Soil, 282, 135-151.

Mo JM, Zhang W, Zhu WX, Fang YT, Li DJ, Zhao P (2007). Response of soil respiration to simulated $\mathrm{N}$ deposition in a disturbed and a rehabilitated tropical forest in southern China. Plant and Soil, 296, 125-135.

Mo JM, Zhang W, Zhu WX, Gundersen P, Fang YT, Li DJ, Wang $H$ (2008). Nitrogen addition reduces soil respiration in a mature tropical forest in southern China. Global Change Biology, 14, 403-412.

Moscatelli MC, Lagornarsino A, de Angelis P, Grego S (2008). Short and mediumterm contrasting effects of nitrogen fertilization on $\mathrm{C}$ and $\mathrm{N}$ cycling in a poplar plantation soil. Forest Ecology and Management, 255, 447-454.

Pendall E, Bridgham S, Hanson PJ, Hungate B, Kicklighter DW, Johnson DW, Law BE, Luo YQ, Megonigal JP, Olsrud M, Ryan MG, Wan SQ (2004). Belowground process responses to elevated $\mathrm{CO}_{2}$ and temperature: A discussion of observations, measurement methods, and models. New Phytologist, 162, 311-322.

Peng SS, Piao SL, Wang T, Sun JY, Shen ZH (2009). Temperature sensitivity of soil respiration in different ecosystems in China. Soil Biology \& Biochemistry, 41, 10081014.

Pregitzer KS, Burton AJ, Zak DR, Talhelm AF (2008). Simulated chronic nitrogen deposition increases carbon storage in northern temperate forests. Global Change Biology, 14, 142-153.

Raich JW, Potter CS, Bhagawati D (2002). Interannual variability in global soil respiration, 1980-1994. Global 
Change Biology, 8, 800-812.

Rodeghiero M, Cescatti A (2006). Indirect partitioning of soil respiration in a series of evergreen forest ecosystems. Plant and Soil, 284, 7-22.

Rühling Å, Tyler G (1991). Effects of simulated nitrogen deposition to the forest floor on the macrofungal flora of a beech forest. Ambio, 20, 261-263.

Ryan MG, Law BE (2005). Interpreting, measuring, and modeling soil respiration. Biogeochemistry, 73, 3-27.

Schlesinger WH, Andrews JA (2000). Soil respiration and the global carbon cycle. Biogeochemistry, 48, 7-20.

Sotta ED, Meir P, Malhi Y, Nobre AD, Hodnett M, Grace J (2004). Soil $\mathrm{CO}_{2}$ efflux in a tropical forest in the central Amazon. Global Change Biology, 10, 601-617.

Sun ZZ, Liu LL, Ma YC, Yin GD, Zhao C, Zhang Y, Piao SL (2014). The effect of nitrogen addition on soil respiration from a nitrogen-limited forest soil. Agricultural and Forest Meteorology, 197, 103-110.

Tang XL, Liu SG, Zhou GY, Zhang DQ, Zhou CY (2006). Soil-atmospheric exchange of $\mathrm{CO}_{2}, \mathrm{CH}_{4}$, and $\mathrm{N}_{2} \mathrm{O}$ in three subtropical forest ecosystems in southern China. Global Change Biology, 12, 546-560.

Tarnocai C, Canadell JG, Schuur EAG, Kuhry P, Mazhitova G, Zimov S (2009). Soil organic carbon pools in the northern circumpolar permafrost region. Global Biogeochemical Cycles, 23, GB2023. doi: 10.1029/2008GB003327.

Thomas RQ, Canham CD, Weathers KC, Goodale CL (2010). Increased tree carbon storage in response to nitrogen deposition in the US. Nature Geoscience, 3, 13-17.

Tu LH, Hu TX, Zhang J, Li XW, Hu HL, Liu L, Xiao YL (2013). Nitrogen addition stimulates different components of soil respiration in a subtropical bamboo ecosystem. Soil Biology \& Biochemistry, 58, 255-264.

Wu ZY (1980). Vegetation of China. Science Press, Beijing. (in Chinese) [吴征镒 (1980). 中国植被. 科学出版社, 北 京.]

Xu WH, Wan SQ (2008). Water- and plant-mediated responses of soil respiration to topography, fire, and nitrogen fertilization in a semiarid grassland in northern China. Soil Biology \& Biochemistry, 40, 679-687.

Zheng SW, Tang M, Zou JH, Mu CL (2007). Summary of research on shrub biomass in China. Journal of Chengdu University (Natural Science Edition), 26, 189-192. (in Chinese with English abstract) [郑绍伟, 唐敏, 邹俊辉, 慕长龙 (2007). 灌木群落及生物量研究综述. 成都大学 学报(自然科学版), 26, 189-192.]

Zheng XH, Fu CB, Xu XK, Yan XD, Huang Y, Han SH, Hu F, Chen GX (2002). The Asian nitrogen cycle case study. AMBIO, 31, 79-87.

Zheng ZM, Yu GR, Fu YL, Wang YS, Sun XM, Wang YH (2009). Temperature sensitivity of soil respiration is affected by prevailing climatic conditions and soil organic carbon content: A trans-China based case study. Soil Biology \& Biochemistry, 41, 1531-1540.

Zhou LY, Zhou XH, Zhang BC, Lu M, Luo YQ, Liu LL, Li B (2014). Different responses of soil respiration and its components to nitrogen addition among biomes: A metaanalysis. Global Change Biology, 20, 2332-2343.

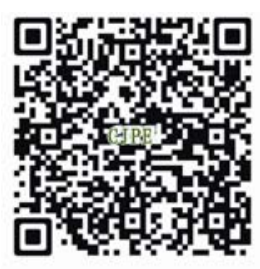

植物生态学报官网

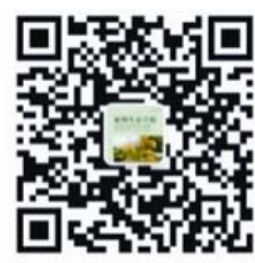

微信订阅号

期刊及学科

相关信息发布
特邀编委: 陈 槐 责任编辑: 王 藏

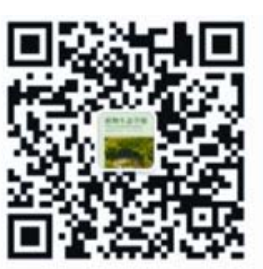

微信服务号

稿件状态查询

全文检索汶览 